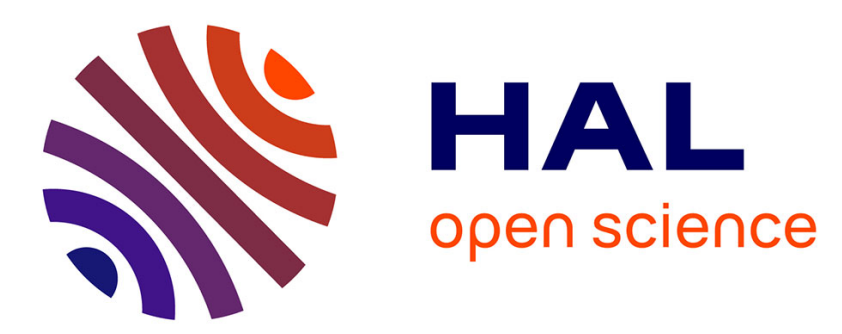

\title{
Calcul du spectre acoustique d'un jet libre subsonique soumis à une injection de masse
}

\author{
V. Moriniere, Y. Gervais
}

\section{To cite this version:}

V. Moriniere, Y. Gervais. Calcul du spectre acoustique d'un jet libre subsonique soumis à une injection de masse. Journal de Physique IV Proceedings, 1994, 04 (C5), pp.C5-951-C5-954. 10.1051/jp4:19945208 . jpa-00252892

\section{HAL Id: jpa-00252892 https://hal.science/jpa-00252892}

Submitted on 1 Jan 1994

HAL is a multi-disciplinary open access archive for the deposit and dissemination of scientific research documents, whether they are published or not. The documents may come from teaching and research institutions in France or abroad, or from public or private research centers.
L'archive ouverte pluridisciplinaire HAL, est destinée au dépôt et à la diffusion de documents scientifiques de niveau recherche, publiés ou non, émanant des établissements d'enseignement et de recherche français ou étrangers, des laboratoires publics ou privés. 


\title{
Calcul du spectre acoustique d'un jet libre subsonique soumis à une injection de masse
}

\author{
V. MORINIERE et Y. GERVAIS
}

Laboratoire d'Etudes Aérodynamiques, 40 Av. du Recteur Pineau, 86022 Poitiers cedex, France

\begin{abstract}
We give a model of the acoustic radiation field of a cylindrical free jet. This model is based on Lighthill's equation with a simplified source term. The theoretical expression of the radiated acoustic field includes the coherence of acoustic sources present in the jet. That means that a normalized cross-spectral density according to a convected Gaussian form function of correlation is assumed for the source quantity. We define then the coherence parameters of this function which are introduced in the computation. As this values can't be determined from theoretical considerations, they are derived from experimental investigations. The sound power spectrum and level of a turbulent free jet in interaction with a injection wreath of cold air are then computed.
\end{abstract}

\section{INTRODUCTION}

La turbulence d'un jet qui se détend dans un milieu au repos est sa principale source de bruit. Elle est fortement cohérente et par conséquent les sources acoustiques qui lui sont associées le sont également. Cette cohérence est prise en compte pour la détermination du contenu spectral et du niveau global de l'émission sonore du jet, avec ou sans injection d'air secondaire latérale.

On considère donc un jet libre subsonique de fluide incompressible et isotherme se développant suivant l'axe $O x$ dans une atmosphère au repos. Le jet est axisymétrique, de telle sorte que le problème est traité en coordonées cylindriques.

Le calcul développé ici repose sur les résultats de Michalke concernant le rôle des structures cohérentes dans un jet libre [1]. Michalke montre que la cohérence d'une quantité turbulente peut être exprimée à partir de la densité interspectrale de forme gaussienne convectée des composantes turbulentes du terme source simplifié du tenseur de Lighthill. Ceci exclue toutes les sources de bruit autres que celles associées à la turbulence du jet, et permet de plus de ne considérer que le bruit issu des zones de mélange et de transition du jet.

Dans la situation considérée, on a trois échelles de longueurs de cohérence $\mathrm{L}_{c x}, \mathrm{~L}_{\mathrm{cr}}$ et $\mathrm{L}_{\mathrm{c} \Phi}$ dans les directions axiale, radiale et azimutale. L'analyse du champ de pression turbulente par transformée de Fourier montre l'existence de modes axiaux, radiaux et azimutaux dans des proportions équivalentes [2]. Toutefois, compte tenu du fait que seuls les modes azimutaux d'ordre 0 et 1 apparaîssent effectivement énergétiques, on détermine l'émission sonore du jet en champ lointain dans n'importe quelle direction, en ne modulant le rayonnement acoustique associé à la zone turbulente la plus énergétique que par des fonctions de cohérence axiale et radiale.

\section{DENSITE SPECTRALE DE PUISSANCE ACOUSTIQUE}

Compte tenu de la nature du jet, les hypothèses de l'analogie de Lighthill sont vérifiées (vitesse du son $c_{0}$ et masse volumique $\rho_{0}$ constantes), et permet ainsi d'estimer le bruit rayonné par les régions turbulentes de l'écoulement [3].

Comme nous l'avons vu, le calcul de la pression acoustique pour un jet circulaire peut être effectué en considérant les deux échelles de cohérence $\mathrm{L}_{\mathrm{cx}}$ et $\mathrm{L}_{\mathrm{cr}}$ dans les directions axiale et radiale. On introduit 
alors deux paramètres de cohérence $\sigma_{\mathrm{X}}$ et $\sigma_{\mathrm{r}}$, définis comme le rapport de l'échelle de cohérence par l'échelle d'extension spatiale du volume source. Ces paramètres sont des fonctions du nombre de Strouhal et du nombre de Mach.

\subsection{Terme source}

Lighthill montre que la pression acoustique rayonnée en champ lointain en un point $\mathrm{x}_{\mathrm{i}}$ par une région source finie est donnée par :

$$
p\left(x_{i}, t\right)=\frac{1}{4 \pi\left|x_{i}\right|} \frac{1}{c_{0}^{2}} \int d y_{i} \frac{\delta^{2} Q\left(y_{i}, t-\tau, x_{i}\right)}{\delta t^{2}}
$$

où $\tau$, défini par $\tau=\frac{\left|x_{i}\right|-x_{i} y_{i} /\left|x_{i}\right|}{c_{0}}$ est le temps de retard,

$x_{i}$ et $y_{i}$ sont les positions respectives du point d'observation en champ lointain et du point source, et $\mathrm{Q}\left(\mathrm{y}_{\mathrm{i}}, \mathrm{t}, \mathrm{x}_{\mathbf{i}}\right)=\mathrm{v}_{\mathbf{i}} \mathbf{v}_{\mathbf{j}} \frac{\mathrm{x}_{\mathbf{i}} \mathrm{x}_{\mathbf{j}}}{\left|\mathrm{x}_{\mathbf{i}}\right|^{2}}$ le terme de source dominant dans un jet isentropique.

On obtient le spectre de puissance acoustique $W_{\mathrm{pp}}$ à partir de l'expression de la pression acoustique donnée par l'équation (1), sur laquelle on effectue une transformée de Fourier :

$$
W_{p p}=\frac{k^{4}}{\left(4 \pi \mid x_{i}\right)^{2}} \int d y_{i 1} \int d y_{i 2} W_{Q_{1} Q_{2}}\left(y_{i 1}, y_{i 2}\right) \exp \left(\tau-\frac{i k x\left(y_{i 2}-y_{i 1}\right)}{\left|x_{i}\right|}\right)
$$

où $k=\omega / c_{0}$ est le nombre d'onde acoustique, $W_{Q_{1} Q_{2}}=Q_{\omega}\left(y_{i 1}\right) Q_{\omega}\left(y_{i 2}\right) f\left(y_{i 2}, y_{i 1}\right)$ est la densité interspectrale de puissance de la quantité source $Q$ entre deux points sources $y_{i 1}$ et $y_{i 2}, Q_{\omega}$ étant la densité spectrale de la quantité source $Q$ et $f$ la fonction de cohérence du terme source $Q$ entre deux points yil et yi2.

Pour décrire la corrélation spatiale de la turbulence dans le jet, on choisit une fonction de corrélation $\mathrm{R}$ de forme gaussienne convectée pour la quantité souce $Q$ entre deux points $\mathrm{y}_{\mathrm{i} 1}$ et $\mathrm{y}_{\mathrm{i} 2}$ :

$$
R=\widetilde{Q}\left(y_{i 1}\right) \widetilde{Q}\left(y_{i 2}\right) \exp \left(-a^{2}\left[\left(x_{2}-x_{1}+U_{c} \tau\right)^{2}+\beta^{2} \tau^{2}\right]-b^{2}\left(r_{2}-r_{1}\right)^{2}\right)
$$

où $\widetilde{Q}\left(y_{i 1}\right)$ est la valeur efficace de la quantité source $Q$ au point yil, et $\beta$ une grandeur liée à la convection.

Ainsi, à partir de la transformée de Fourier du coefficient de corrélation $\mathrm{R}$, on obtient la fonction de cohérence $f$ de la quantité source $Q$ entre deux points yi1 et $y_{i 2}$. On a :

$$
\mathrm{W}_{\mathrm{Q}_{1} Q_{2}}=\int_{0}^{+\infty} R \mathrm{e}^{\mathrm{i} \omega \tau} \mathrm{d} \tau
$$

soit :

$$
\mathrm{W}_{\mathrm{Q}_{1} \mathrm{Q}_{2}}=\mathrm{Q}_{\omega}\left(\mathrm{y}_{\mathrm{i} 1}\right) \mathrm{Q}_{\omega}\left(\mathrm{y}_{\mathrm{i} 2}\right) \exp \left(-\mathrm{A}^{2}\left(\mathrm{x}_{2}-\mathrm{x}_{1}\right)^{2}+\mathrm{i} \alpha\left(\mathrm{x}_{2}-\mathrm{x}_{1}\right)-\mathrm{b}^{2}\left(\mathrm{r}_{2}-\mathrm{r}_{1}\right)^{2}\right)
$$

ce qui conduit à :

$$
f\left(y_{i 1}, y_{i 2}\right)=\exp \left(-A^{2}\left(x_{2}-x_{1}\right)^{2}+i \alpha\left(x_{2}-x_{1}\right)-b^{2}\left(r_{2}-r_{1}\right)^{2}\right)
$$

où $\mathrm{A}$ et $\mathrm{b}$ sont des fonctions du nombre de Strouhal.

\subsection{Echelles de longueur}

Le calcul de la puissance acoustique rayonnée nécessite la définition des échelles de longueur de cohérence axiale $L_{c x}$ et radiale $L_{c r}$. Ceci est effectué à partir de l'équation (4), et donne :

$$
L_{c x}=\frac{\sqrt{\pi}}{A} \quad \text { et } \quad L_{c r}=\frac{\sqrt{\pi}}{b}
$$

Pour simplifier l'analyse, on suppose que la répartition spatiale des sources dans le jet est de la forme suivante : 


$$
Q_{\omega}\left(y_{i}\right)=Q_{\omega_{\max }} q_{x}(x) q_{r}(r)
$$

où les fonctions de répartition des sources $\mathrm{q}_{\mathrm{x}}$ et $\mathrm{q}_{\mathrm{r}}$ sont également de forme gaussienne. Cette hypothèse permet de ne considérer que le bruit de mélange, en considérant $\mathrm{q}_{\mathrm{x}}$ et $\mathrm{q}_{\mathrm{r}}$ centrées sur ces zones. On peut alors définir des échelles de longueur de source axiale $\mathrm{L}_{\mathrm{sx}}$ et radiale $\mathrm{L}_{\mathrm{sr}}$ :

$$
L_{s x}=\int_{0}^{+\infty} q_{x}(x) d x \quad L_{s r}=2 \pi \int_{0}^{+\infty} q_{r}(r) d r
$$

\subsection{Expression du spectre acoustique}

En introduisant les relations (4) à (7) dans l'équation (2), on obtient le spectre de bruit de jet :

$$
\mathrm{W}_{\mathrm{pP}}=\pi^{2}\left(\frac{\mathrm{D}}{\left|\mathrm{x}_{\mathrm{i}}\right|}\right)^{2} \mathrm{M}^{4} S \mathrm{t}^{4} \mathrm{Q}_{\omega_{\mathrm{m}}}^{2}\left(\frac{\mathrm{L}_{\mathrm{sx}}}{\mathrm{D}}\right)^{4}\left(\frac{\mathrm{L}_{\mathrm{sr}}}{\mathrm{D}}\right)^{2} \mathrm{G}_{\mathrm{x}}\left(\sigma_{\mathrm{x}}, \mathrm{C}\right) \mathrm{G}_{\mathrm{x}}\left(\sigma_{\mathrm{r}}, \delta\right)
$$

où les expressions des fonctions de cohérence axiale et radiale $G_{\mathbf{x}}$ et $G_{\mathbf{r}}$ sont les suivantes :

$$
G_{x}=\frac{\sigma_{x}}{\sqrt{2+\sigma_{x}^{2}}} \exp \left(-\frac{C^{2} \sigma_{x}^{2}}{2 \pi\left(2+\sigma_{x}^{2}\right)}\right) \quad \text { et } \quad G_{x}=\frac{\sigma_{x}}{2+\sigma_{x}^{2}} \exp \left(-\frac{\delta^{2} \sigma_{x}^{2}}{2 \pi\left(2+\sigma_{x}^{2}\right)}\right)
$$

avec $\mathrm{D}$ diamètre du jet, $\mathrm{M}$ nombre de $\mathrm{Mach}, \mathbf{S t}$ nombre de Strouhal, $\delta$ un paramètre d'épaisseur acoustique du jet et $C$ un paramètre de convection. Les paramètres de cohérence $\sigma_{\mathrm{x}}$ et $\sigma_{\mathrm{r}}$ sont définis par :

$$
\sigma_{\mathrm{x}}=\frac{\mathrm{L}_{\mathrm{cx}}}{\mathrm{L}_{\mathrm{ax}}} \quad \text { et } \quad \sigma_{\mathrm{x}}=\frac{\mathrm{L}_{\mathrm{cr}}}{\mathrm{L}_{\mathrm{xr}}}
$$

L'expression théorique du champ acoustique rayonné par une composante fréquentielle peut alors être calculé numériquement.

\section{CAlCUl NUMERIQUe et discussions}

\subsection{Développements numériques}

Le problème le plus difficile à résoudre dans ce calcul concerne la valeur à attribuer aux différentes échelles de longueur définies précédemment. Le modèle utilisé repose sur les mesures expérimentales de Fuchs et Michel [4], qui montrent que l'échelle de longueur axiale $L_{s x}$ est de la forme : $L_{s x}=\frac{U c}{f}$. Cette relation traduit une décroissance inversement proportionnelle à la fréquence de l'échelle intégrale de turbulence dans les zones de mélange et de transition du jet. D'autre part, les mesures donnent un rapport d'environ $1 / \pi$ entre les échelles axiale et radiale. On a donc $L_{s x}=\frac{L_{s \pi}}{\pi}$.

Le développement axiale de la région cohérente est défini par $: L_{c x}=2 D$. Goldstein indique que la longueur de cohérence longitudinale des tourbillons dans la direction de l'écoulement est envìron deux fois celle dans la direction radiale dans la zone de mélange [5]. On a donc $\mathrm{L}_{\mathrm{cr}}=\frac{\mathrm{L}_{\mathrm{cr}}}{2}$.

En pratique, les grandeurs turbulentes nécessaires au calcul du spectre acoustique du jet sont issues d'un code de calcul basé sur le modèle $(k-\varepsilon)$. La détermination du maximum de la quantité source $Q$ est effectuée en recherchant le maximum d'intensité de turbulence situé dans les zones de mélange et de transition du jet. En effet, en dehors de ces zones, c'est à dire pour $x / D>10$, on considère que le rayonnement acoustique est négligeable.

\subsection{Résultats}

Dans un premier temps on calcule l'émission sonore d'un jet libre turbulent, puis on effectue une injection d'air froid avec une couronne circulaire. Pour un jet libre subsonique, on obtient un spectre acoustique large bande, avec une fréquence maximum d'émission correspondant à un nombre de Strouhal égal a 0.23, ce qui est conforme aux résultats expérimentaux (Fig. 1). Les résultats numériques montrent qu'une injection d'air froid à faible vitesse dans un jet d'air froid subsonique ne modifie pas le contenu du spectre acoustique du jet seul, mais agit globalement sur l'amplitude (Fig. 2). 
On calcule également les niveaux globaux de puissance acoustique rayonnée par les configurations précédentes pour différents angles d'observation, ainsi que la puissance acoustique globale émise par un jet seul puis par un jet subsonique en interaction avec une couronne d'injection d'air. Là encore, les résultats thériques obtenus sont conformes à l'experience.

Ce modèle permet ainsi de prévoir globalement les fréquences maximales des spectres de puissance acoustique rayonnés par les zones de mélange et de transition d'un jet libre et d'un jet en interaction avec une couronne d'injection d'air froid.

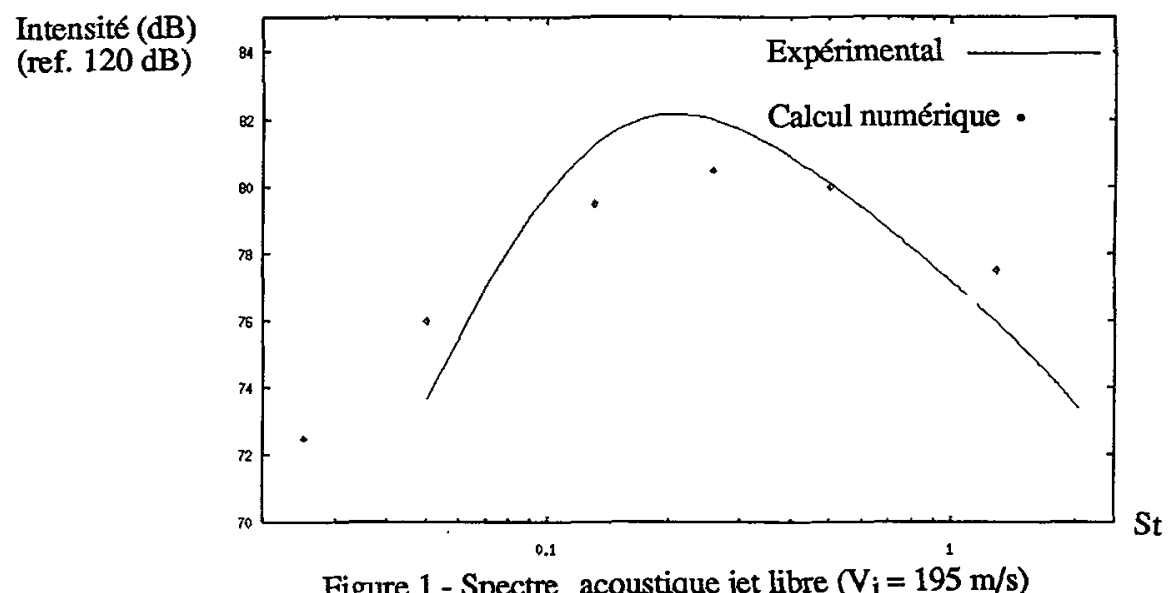

Intensité (dB) (ref. $120 \mathrm{~dB}$ )

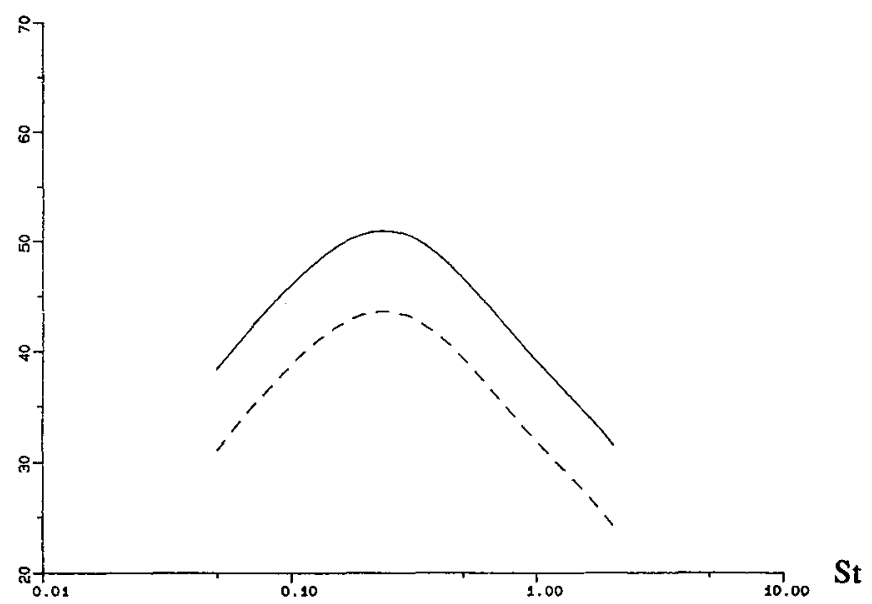

Figure 2 - Spectres acoustiques sans et avec injection secondaire $\left(V_{j}=50 \mathrm{~m} / \mathrm{s}\right)$ sans injection ; - -.....- avec injection $\left(\frac{\text { qinj }_{\text {qjet }}}{q_{\text {jet }}}=10\right.$ )

[1] MICHALKE, A., J.of Sound and Vibration (1977), 55(3), pp 377-394

[2] MICHALKE, A., J.of Sound and Vibration (1983), 8(1), pp 1-17

[3] LIGHTHILL, M.J., Proc.Roy.Soc.(1952), A211, pp 564-587

[4] FUCHS, H.V. and MICHEL,U., AIAA 4th Aeroacoustics Conf. Atlanta, Georgia (1977)

[5] GOLDSTEIN, M.E., Aeroacoustics, Mc Graw Hill Int. Book Compagny (1976) 